\title{
RDWG technique of determination of moisture content in Oil Palm Fruits
}

\author{
Z. Abbas ${ }^{1,2, a}$, R. Mokhtar ${ }^{3}$, K. Khalid ${ }^{2}$, M. Hashim², and S. Abdul Aziz ${ }^{2}$ \\ 1 INSPEM, Universiti Putra Malaysia, 43400 UPM Serdang, Malaysia \\ 2 Department of Physics, Universiti Putra Malaysia, 43400 UPM Serdang, Malaysia \\ 3 College of Engineering, Universiti Tenaga Nasional, 43009 Kajang, Malaysia
}

Received: 23 January 2007 / Accepted: 29 June 2007

Published online: 1 November 2007 - (C) EDP Sciences

\begin{abstract}
Both the reflection and transmission coefficients of oil palm fruits of various moisture contents have been measured using a rectangular dielectric waveguide (RDWG) technique in the frequency range between $8 \mathrm{GHz}$ and $12 \mathrm{GHz}$. Good agreement between predicted and measured values of reflection coefficient suggests the potential of RDWG technique as a new, fast and accurate method for the determination of moisture content in oil palm fruits.
\end{abstract}

PACS. 84.40.-x Radiowave and microwave (including millimeter wave) - 41.20.Jb Electromagnetic wave propagation; radiowave propagation -84.40 . Az Waveguides, transmission lines, striplines

\section{Introduction}

A rectangular dielectric waveguide (RDWG) technique has been used to determine the complex permittivity of materials in the frequency range between $26 \mathrm{GHz}$ and $110 \mathrm{GHz}[1-3]$. The RDWG technique is an efficient, flexible and accurate technique to measure both the effective refractive index and the actual permittivity of samples with cross-sectional dimension as as small as standard rectangular waveguide dimension. However, the technique has only been reported for low loss factor and low dielectric constant materials.

This paper presents an extension of the RDWG technique to determine the variation in both the reflection coefficient and transmission coefficient with frequency at various percentages of moisture content in oil palm fruits.

An oil palm fruit is a lossy, high dielectric constant material. Its main constituents are oil, water and fibre. An oil palm fruit bunch consists of hundreds of fruits. Up to approximately 14-15 weeks after anthesis, the amount of moisture content in fresh mesocarp of the fruit is about $80 \%$ and decreases rapidly to about $30-40 \%$ in the ripe fruit at 20-30 weeks after anthesis. Therefore, a simple, fast and accurate method for the determination of moisture content in the oil palm fruits is important for assessing the quality of fruits that reach the factory.

\footnotetext{
a e-mail: za@f sas.upm.edu.my
}

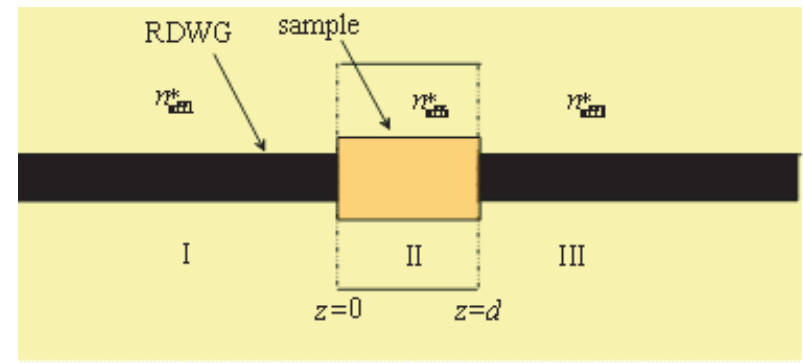

Fig. 1. Effective Index Model of RDWG and sample.

\section{Theory}

Consider the sample as a double step discontinuity in an open dielectric waveguide as shown in Figure 1. The effective index model has been proposed [1-3] to represent the dielectric waveguides and sample with their surrounding (air) as homogenous media with effective refractive indices $n_{\text {eff } 1}$ and $n_{\text {eff } 2}$, respectively.

The complex transmission coefficient $T$ and reflection coefficient $R$ due to the RDWG/sample/RDWG interfaces and multiple reflections were considered as effective values which included both the surface and continuous waves. For samples with low loss, low dielectric constant materials of sufficiently large cross-section the permittivity equals the square of the effective refractive index, $\varepsilon *=n_{\text {eff }}^{2}$ whilst the calculation of both $T$ and $R$ takes the form of the familiar 
plane wave calculation for unbounded homogeneous media

$$
\begin{aligned}
& R=\frac{\left(1-P^{2}\right) \Gamma}{1-\Gamma^{2} P^{2}} \\
& T=\frac{\left(1-\Gamma^{2}\right) P}{1-\Gamma^{2} P^{2}}
\end{aligned}
$$

where $\Gamma$ is the reflection coefficient due to the sample at the boundaries $z=0$ and $z=d$ and the propagation factor $P$ of the sample of thickness $d$ due to propagation constant $\gamma_{s}$ is $P=\exp \left(-\gamma_{\mathrm{S}} d\right)$. The propagation constant can be determined once the complex permittivity $\varepsilon^{*}$ of the sample is known using $\gamma_{s}=k_{0} \sqrt{\varepsilon^{*}}$ where $k_{0}$ is the free space number. The calculation of $T$ and $R$ using equations (1) and (2) is straight forward for a sample with lossy, high dielectric constant material if the sample's cross section equals or larger than the transverse dimension of the RDWG.

In this work, the dielectric mixture model was used to calculate the permittivity for given moisture content values based on the relationship [4]

$$
\sqrt{\varepsilon^{*}}=v_{w} \sqrt{\varepsilon_{w}^{*}}+v_{f} \sqrt{\varepsilon_{f}^{*}}+v_{o} \sqrt{\varepsilon_{o}^{*}}
$$

where $v_{w}, v_{f}$ and $v_{o}$ are the volume fractions of water, fiber and oil, respectively and $\varepsilon_{w}^{*}, \varepsilon_{f}^{*}$ and $\varepsilon_{o}^{*}$ are the corresponding complex permittivities. The values of $\varepsilon_{w}^{*}$ are obtained from the Cole-Cole model [5].

\section{Materials and methods}

\subsection{Sample preparation}

Twenty bunches of tenera variety from different 12 years old oil palm tree were chosen from the university farm for this study. Sampling was done on fruits of various degree of ripeness. To avoid interfering with the normal maturation process, the proper choice of sampling would be from the outer fruits around the equatorial of the bunch. Abnormal, not fully developed, dry and rotten fruits were not considered. The surface of each fruits was wiped dry to clear excess surface moisture. The fresh part of the fruit mesocarp was separated from the nut and cut into small pieces. Then, the small pieces were crumbled to form semi-solid sample. The sample was then placed in a sample holder. Approximately thirteen to fifteen fruits were required to fill the sample holder completely. Fruits were grouped according to colors. More than 500 fruits samples were selected allowing batch sampling ranging from $30 \%$ to $84 \%$ moisture content.

After microwave measurements, the amount of moisture content for each batch of fruit samples was determined using PORIM standard test method [6]. Sample moisture measurements were done in duplicates to ensure accurate result. First, the petri dish with its lid was weighed and left in the dessicator at $26^{\circ} \mathrm{C}$ for a minimum of $30 \mathrm{~min}$. Then, the fruit sample was spread uniformly over the whole base of the dish and re-weighed. These operations must be done as quickly as possible to avoid any

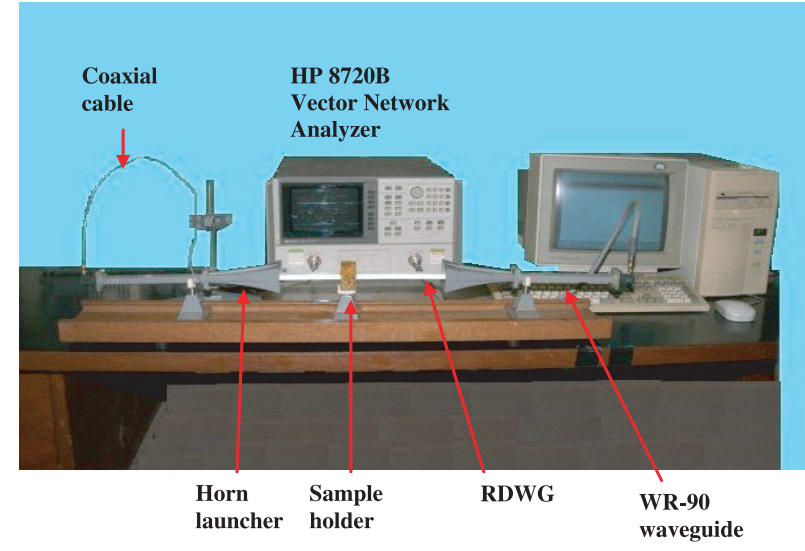

Fig. 2. Measurement Setup.

appreciable change in moisture content. The dish with the fruit sample was place in an oven set at $103 \pm 2{ }^{\circ} \mathrm{C}$ with the lid taken off. Initially, the sample was dried for two hours. The dish was transferred to the dessicator and weighed as soon as it has cooled to laboratory temperature. The process of moisture determination is considered complete if the difference between the two weighing was equal or less than $0.005 \mathrm{~g}$. Else, the sample was subjected to successive $1 \mathrm{~h}$ periods in the oven until the difference in the two consecutive weighings is less than or equal to $0.005 \mathrm{~g}$.

\section{Experimental set-up}

The microwave measurement setup as shown in Figure 2 consists of a HP8720B Vector Network Analyzer (VNA), RDWG, WR-90 standard waveguides, horn antennas, RDWG and a $24 \mathrm{~mm}$ thick sample holder made of polyethylene with cross-sectional dimension $30 \mathrm{~mm} \times$ $50 \mathrm{~mm}$. All microwave measurements were carried out using the VNA in the frequency range between $8 \mathrm{GHz}$ and $12 \mathrm{GHz}$. The sample holder has two openings for the RDWG with cross-sectional dimension equal to WR-90 waveguide size allowing direct contact between RDWG/sample/RDWG. The RDWG was constructed from polytetrafluoroethylene (PTFE) with permittivity $\varepsilon^{*}=2.04-j 0.0006$. PTFE was chosen as the RDWG material due to easy fabrication, very low loss and low dielectric constant properties. The low dielectric constant of PTFE provides a wide coverage of single $E_{11}^{y}$ mode.

The RDWG was made slightly larger than the standard WR-90 waveguide to ensure no air gap at the transition between the RDWG and WR-90 waveguide. The feed end of the RDWG was tapered to reduce reflection between the RDWG and the standard waveguide. Only the $\mathrm{H}$-plane of the RDWG was tapered, to allow natural transition from LSE mode to the $\mathrm{TE}_{10}$ mode, i.e. from centerloaded, partially dielectric-filled to completely dielectricfilled waveguide.

A two stage calibration of the VNA was implemented in this work. First, a full two port calibration for transmission and reflection measurements of two-port devices was implemented at the coaxial cable ends using the 


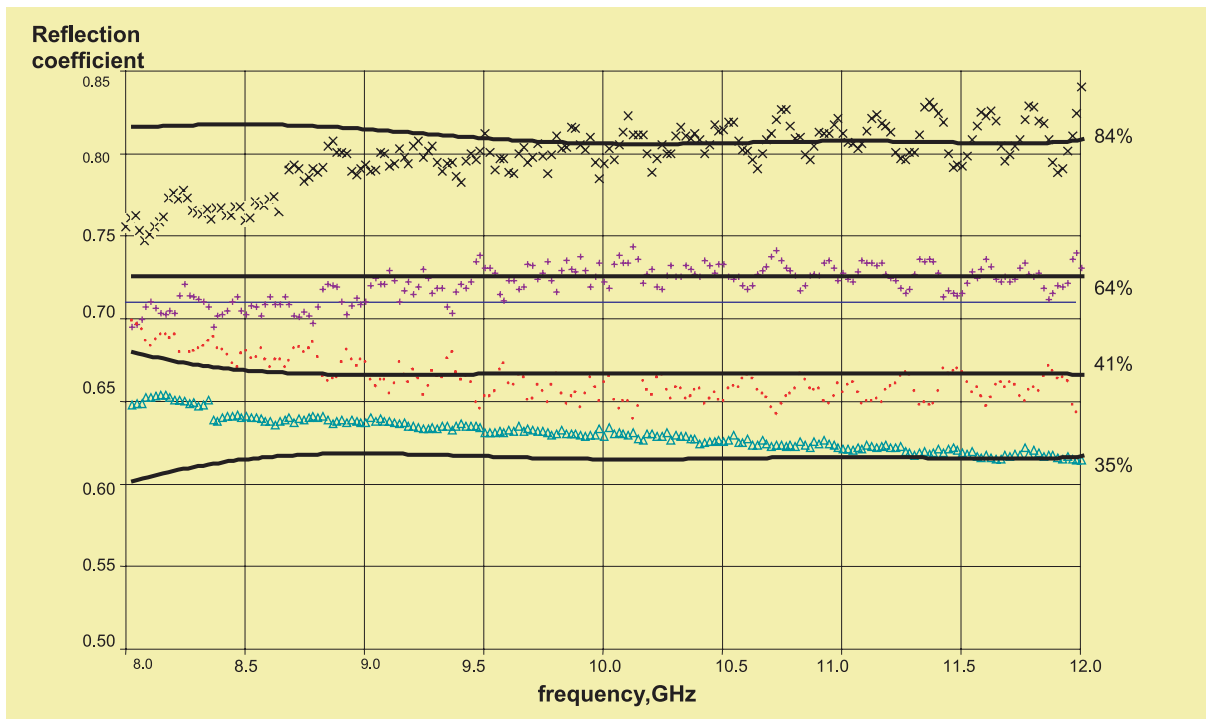

(a)

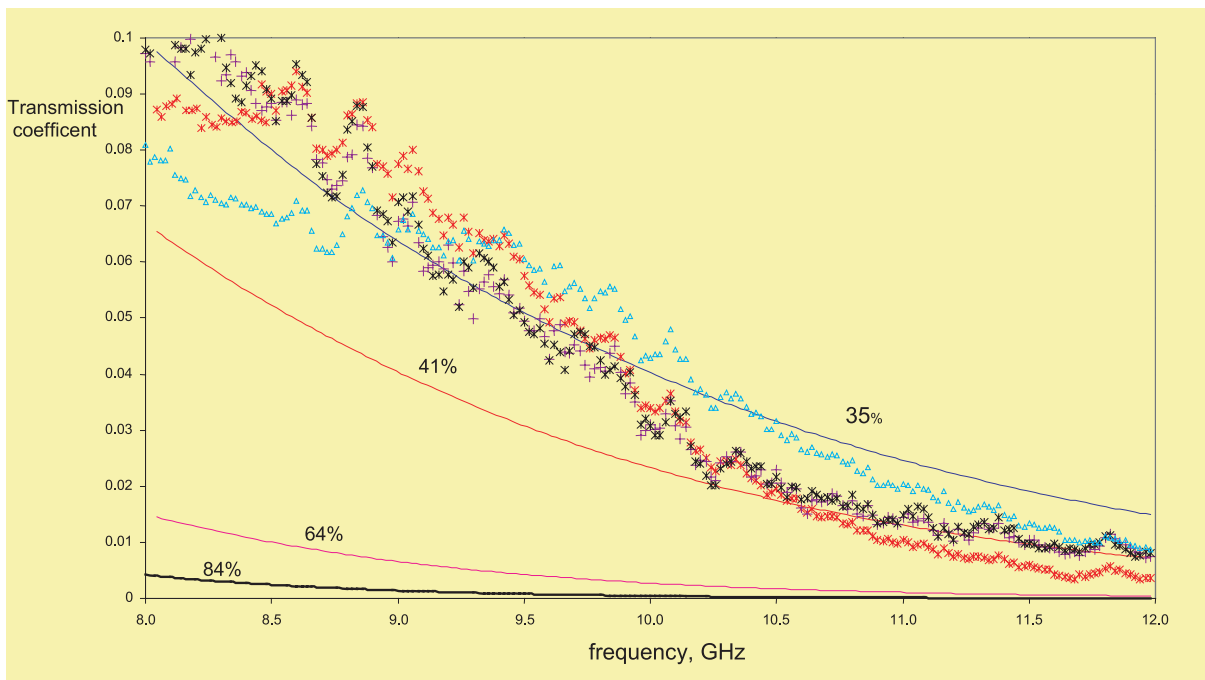

(b)

Fig. 3. Variation in (a) reflection coefficient (b) transmission coefficient with frequency. $(\times 84 \%+64 \% 041 \% \Delta 35 \%)$

VNA calibration standards. The objective was to minimize the VNA measurements errors due to directivity, source match, load match, isolation and frequency response, in both forward and reverse directions. In the second stage of the calibration procedure, the calibration plane was extended to the RDWG ends by applying frequency response error correction technique. The zerolength through standard was implemented by defining the calibration plane as the plane of direct contact between the two RDWG. This is especially advantageous because it does not require reference plane rotation from the calibration plane to the sample ends.

\section{Results and discussions}

The variations in reflection coefficient and transmission coefficient with frequency at various percentages of moisture content for oil palm fruits are illustrated in Figure 3. The line and data points represent the theoretical and measured values, respectively. The mean relative errors between the measured and theoretical values are listed in Table 1 for both the reflection and transmission coefficients for all the moisture content values. The errors were found to be less than $2.5 \pm 0.1 \%$ for the reflection coefficient. Unfortunately very high discrepancy between the measured and calculated transmission coefficient was found especially for high moisture content values. The high deviation is probably due to lack of sensitivity of the VNA for magnitudes less than 0.1. It can be clearly seen that only the reflection coefficients show good agreement between the theoretical and measured values. The results suggest reflection measurements alone are sufficient to provide a unique classification of fruit quality based on the amount of moisture content as illustrated in Figure 4. For example, the mean values of the reflection coefficient 
Table 1. Mean relative errors of reflection and transmission coefficient.

\begin{tabular}{llllll}
\hline & & \multicolumn{4}{c}{$\%$ moisture content } \\
\cline { 3 - 6 } & & 35 & 41 & 64 & 84 \\
\hline \multirow{2}{*}{$\begin{array}{l}\text { \%relative } \\
\text { error }\end{array}$} & reflection & $2.4 \pm 0.1$ & $2.5 \pm 0.1$ & $2.2 \pm 0.1$ & $2.1 \pm 0.1$ \\
\cline { 2 - 6 } & transmission & $18.8 \pm 0.9$ & $7880 \pm 529$ & $1024 \pm 19$ & $9116 \pm 394$ \\
\hline
\end{tabular}

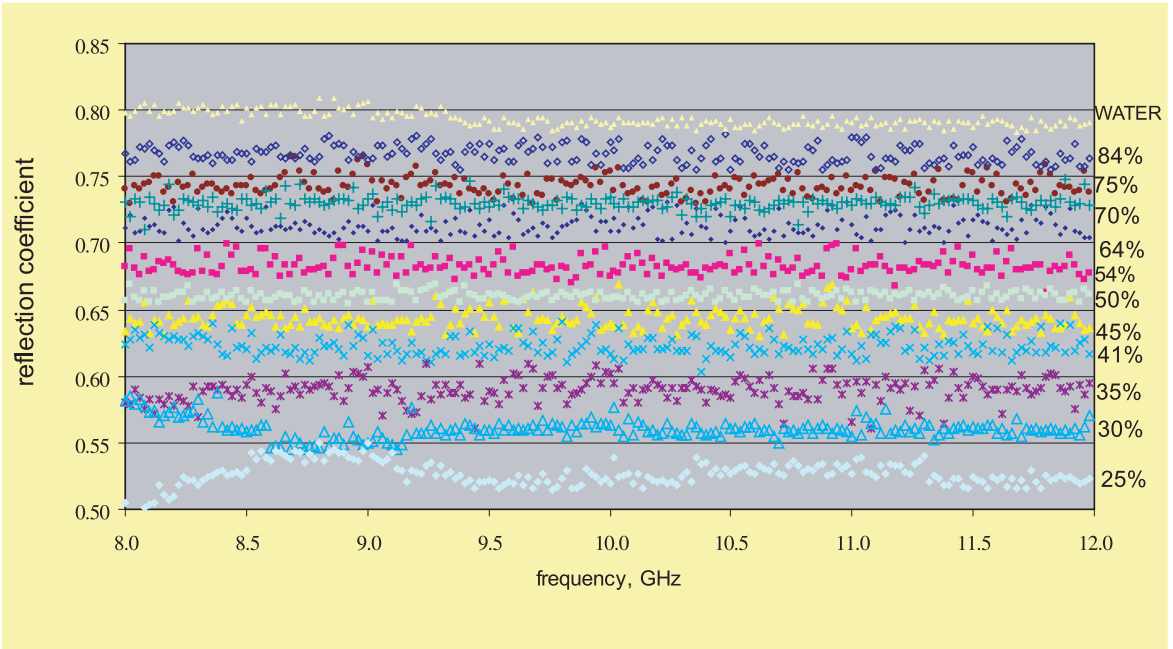

Fig. 4. Variation in reflection coefficient with frequency at various percentages of moisture content.

for ripe fruits (30-40\% m.c.) using RDWG technique is $0.604 \pm 0.001$ in the frequency range between $8 \mathrm{GHz}$ and $12 \mathrm{GHz}$.

The RDWG technique allows a fast and accurate method to determine the amount of moisture content in oil palm fruits. Moisture measurements using the sensor do not offer the accuracy of the time-consuming standard oven drying method but is suitable for a fast, first quality check of fruit ripeness. A portable microwave measurement system shall be constructed in the near future to replace the VNA for in situ measurements.

\section{Conclusions}

A rectangular dielectric waveguide technique (RDWG) has been developed for measurements of both the transmission and reflection coefficient of the oil palm fruits in the frequency range between $8 \mathrm{GHz}$ and $12 \mathrm{GHz}$. The results indicate that RDWG technique can accurately determine the quality of oil palm fruits based only on reflection measurements. The maximum relative error between the measured and predicted reflection coefficient was found to have a mean value of less $2.5 \pm 0.1 \%$.

The authors wish to thank the Ministry of Science, Technology and Environment Malaysia for their support under project number: IRPA 09-02-04-0460-EA001 and the NSF scholarship to Roslina Mokhtar.

\section{References}

1. Z. Abbas, R.D. Pollard, R.W. Kelsall, IEEE Trans. Instrum. Meas. 50, 1334 ( 2001)

2. Z. Abbas, R.D. Pollard, R.W. Kelsall, IEEE Trans. Instrum. Meas. 47, 148 (1998)

3. Z. Abbas, R.D. Pollard, R.W. Kelsall, IEEE Trans. Microwave Theory Tech. 46, 2011(1998)

4. Z. Abbas, Y.K. Yeow, A.H. Shaari, K. Khalid, J. Hassan, E. Saion, IEEE Sensors J. 5, 1281 (2005)

5. E. Nyfors, P. Vainikainen, Industrial Microwave Sensors, (Artech House, Norwood MA, 1989)

6. W.L. Siew, T.S Tang, T.A. Tan, Porim Test Methods (Palm Oil Research Institute of Malaysia, Bangi, 1995) 